\title{
Optimization of extraordinary optical absorption in plasmonic and dielectric structures
}

\section{Dühring, Maria Bayard; Sigmund, Ole}

\section{Published in:}

Optical Society of America. Journal B: Optical Physics

Link to article, DOI:

10.1364/JOSAB.30.001154

Publication date:

2013

Document Version

Publisher's PDF, also known as Version of record

Link back to DTU Orbit

Citation (APA):

Dühring, M. B., \& Sigmund, O. (2013). Optimization of extraordinary optical absorption in plasmonic and dielectric structures. Optical Society of America. Journal B: Optical Physics, 30(5), 1154-1160.

https://doi.org/10.1364/JOSAB.30.001154

\section{General rights}

Copyright and moral rights for the publications made accessible in the public portal are retained by the authors and/or other copyright owners and it is a condition of accessing publications that users recognise and abide by the legal requirements associated with these rights.

- Users may download and print one copy of any publication from the public portal for the purpose of private study or research.

- You may not further distribute the material or use it for any profit-making activity or commercial gain

- You may freely distribute the URL identifying the publication in the public portal 


\title{
Optimization of extraordinary optical absorption in plasmonic and dielectric structures
}

\author{
Maria B. Dühring and Ole Sigmund* \\ Technical University of Denmark, Department of Mechanical Engineering, Lyngby 2800 Kgs., Denmark \\ ${ }^{*}$ Corresponding author: sigmund@mek.dtu.dk
}

Received January 10, 2013; accepted February 18, 2013;

posted March 7, 2013 (Doc. ID 183232); published April 10, 2013

\begin{abstract}
Extraordinary optical absorption (EOA) can be obtained by plasmonic surface structuring. However, studies that compare the performance of these plasmonic devices with similar structured dielectric devices are rarely found in the literature. In this work we show different methods to enhance the EOA by optimizing the geometry of the surface structuring for both plasmonic and dielectric devices, and the optimized performances are compared. Two different problem types with periodic structures are considered. The first case shows that strips of silicon on a surface can increase the absorption in an underlying silicon layer for certain optical wavelengths compared to metal strips. It is then demonstrated that by topology optimization it is possible to generate nonintuitive surface designs that perform even better than the simple strip designs for both silicon and metals. These results indicate that in general it is important to compare the absorption performance of plasmonic devices with similarly structured dielectric devices in order to find the best possible solution. (c) 2013 Optical Society of America

OCIS codes: (240.0310) Thin films; (310.6628) Subwavelength structures, nanostructures.

http://dx.doi.org/10.1364/JOSAB.30.001154
\end{abstract}

\section{INTRODUCTION}

The field of plasmonic structures has developed quickly since the results on extraordinary optical transmission through subwavelength apertures in metal films were published more than a decade ago [1]. The theory behind this has been examined [2], and the extreme light concentration of plasmonic structures has been used in a wide range of applications such as sensors, novel optical lenses, ultrafast and compact photodetectors and modulators, near-field scanning optical microscopy and nonlinear optics [3, $\underline{3}]$. Recently, plasmon excitation and light localization have been used to enhance the optical absorption, the so-called extraordinary optical absorption (EOA), in a number of different thin-film solar cell structures [5-9]. Purely dielectric gratings can also enhance absorption [10]; however, the enhancement factors reported for plasmonic structures are usually based on comparisons with bare dielectric surfaces. An example is found in [8], where a systematic approach is suggested to increase the absorption in a thin Si film over the solar spectrum by distributing Ag strips on top of a $\mathrm{SiO}_{2}$-coated $\mathrm{Si}$ film on a $\mathrm{SiO}_{2}$ substrate. The optical waves pass through the slits between the Ag strips, and localized modes are generated in the Si film. It is shown that the normalized short-circuit current for the solar spectrum is enhanced by more than $40 \%$ compared to a bare dielectric surface. Lately, however, some researchers [11-15] have reported that equal or even better absorption enhancement can be obtained from simple purely dielectric grating surfaces that eliminate thermal losses associated with losses in metallic parts.

In this paper we further challenge the idea of EOA through purely dielectric designs described in more detail. In a previous work [12] the authors studied simple grating structures with strips made from three different metals $(\mathrm{Ag}, \mathrm{Al}$, and $\mathrm{Au})$ and compared them to the performance for $\mathrm{Si}$ and $\mathrm{SiO}_{2}$ strips. The study showed that similar enhancement can be obtained for strips of metals and $\mathrm{Si}$, as similar localized mode effects are generated in the structures. For Si strips, however, less energy is lost in the strips and hence the enhancements are in general greater and less dependent on cell dimensions and wavelength. This leads to a normalized short-circuit current that is four times bigger for the Si strips compared to the metal strips. $\mathrm{SiO}_{2}$ strips only show a slightly better performance compared to a bare surface, because the refractive index contrast is too low to support localized modes. For this particular device a purely dielectric grating outperforms the plasmonic-based counterpart, and we did not observe any of the advantages usually associated with metallic gratings. References [11] and [14] point in the same direction by suggesting use of semiconductor-based nanowires and grating structures instead of plasmonics for similar reasons. We therefore argue that comparisons with bare surfaces are not appropriate when designing plasmonic devices, and in general they have to be compared to similarly structured dielectric devices in order to find the best technological solution.

In the present paper we study the topic in more detail. Again, we approach it by examining two different solar cell problems where the surface structures are composed of either metal or dielectric material. First, the performance is optimized by parameter studies, and subsequently we use topology optimization to find optimal grating profiles for both plasmonic and purely dielectric structures. The problems considered are both based on periodic cells with incident waves normal to the surface. The numerical model is described in Section 2 .

The first problem type is studied in Section 3 and concerns the enhancement of the EOA in a structure where strips of 
either Si or a metal are deposited on the surface of a Si substrate. The structure is inspired by [7], and a parameter study on the size of the strips is performed for the different strip materials for four individual wavelengths in order to increase the absorption in the Si substrate. The results are compared to see if the metals or the $\mathrm{Si}$ are performing the best.

In Section $\underline{4}$ we extend the study of the cell by applying a gradient-based topology optimization method to design surfaces that additionally enhance the EOA. Topology optimization as a computer-based method was originally developed in 1988 in order to maximize the stiffness of structures for a limited amount of material [16]. The method allows air and solid material to be distributed freely in a chosen design domain such that the size, shape, and number of holes are determined. The method therefore offers more freedom in the design than can be obtained by simpler parameter studies. The method was later applied to a range of other engineering fields including mechanism design, heat transfer, and fluid flow problems [17]. During the last decade it has been extended to the field of electromagnetic wave propagation problems and applied to design planar photonic-bandgap materials [18,19], bends, and splitters based on 2D bandgap materials [20-22], optical fibers $[23,24]$ and 2D periodic structures with desirable transmission properties [25]. Topology optimization for plasmonic structures is presented in [26], where grating couplers for the efficient excitation of surface plasmons are designed. Recently, the approach has also been used to design nanosurfaces for structural color generation [27]. Theory and applications of topology optimization within the field of nanophotonics are reviewed in the paper by Jensen and Sigmund [28]. The method therefore proves promising for designing structures that can improve EOA. In the present work a layer on top of the Si layer is used as the design domain, where an optimized distribution of air and solid material (either $\mathrm{Si}$ or $\mathrm{Al}$ ) is determined by topology optimization. The method is employed in order to enhance the structure both for one wavelength and for a number of wavelengths.

\section{NUMERICAL MODEL}

The structures considered in the present work are periodic, and the geometry of the cell with period $p$ is seen in Fig. 1 . The geometry is inspired by the structure found in [7]. The optical wave with amplitude $u_{0}$ is excited along the input boundary $\Gamma_{\mathrm{in}}$. A part of the energy from the optical wave is absorbed in the Si layer $\Omega_{\text {abs }}$, which has thickness $a$. Each end of the cell is terminated by perfectly matched layers (PMLs) [29], and the lower and upper boundaries, $\Gamma_{l}$ and $\Gamma_{u}$, are periodic. The strips on the surface can consist of the four different materials $\mathrm{Si}, \mathrm{Al}, \mathrm{Ag}$, and $\mathrm{Au}$. It is assumed

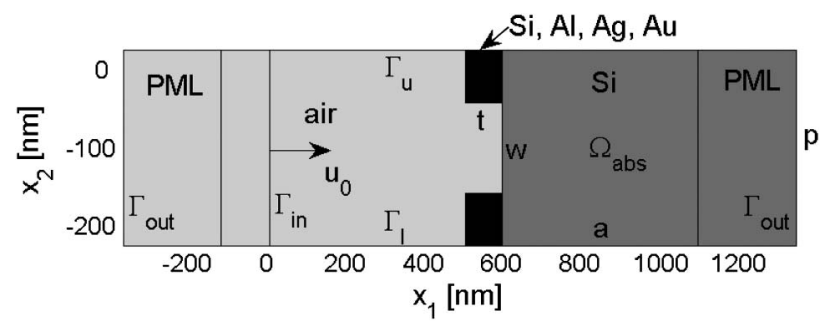

Fig. 1. Periodic cell structure with period $p$. The strips on the surface with thickness $t$ can consist of the four different materials $\mathrm{Si} \mathrm{Al}, \mathrm{Ag}$, and $\mathrm{Au}$. The absorbed energy is measured in $\Omega_{\mathrm{abs}}$, and PMLs are added at each end of the domain. that the problem with the domain $\Omega$ is governed by the scalar Helmholtz equation for time-harmonic waves [30],

$$
\frac{1}{\gamma_{i}} \frac{\partial}{\partial x_{i}}\left(\frac{1}{\gamma_{i}} A \frac{\partial u}{\partial x_{i}}\right)+k_{0}^{2} B u=0 \quad \text { in } \Omega,
$$

where $A=1 / \varepsilon_{r}$ and $B=\mu_{r}$ for $H_{3}$ polarization and $A=1 / \mu_{r}$ and $B=\varepsilon_{r}$ for $E_{3}$ polarization. $\varepsilon_{r}$ is the relative permittivity of the materials. $\mu_{r}$ is the relative permeability and will be equal to 1 in the following. $k_{0}=\omega / c_{0}$ is the propagation constant, $\omega$ is the angular frequency of the optical wave, and $c_{0}=$ $1 / \sqrt{\mu_{0} \varepsilon_{0}}$ is the speed of light in vacuum, with $\mu_{0}$ and $\varepsilon_{0}$ being the permeability and permittivity in vacuum. The parameter $\gamma_{i}$ is an artificial extra damping at the position $x_{i}$ in the PML and has the value 1 in the normal domains. As the PML is added in the $x_{1}$ direction, only $\gamma_{1}$ has a value different from 1 and is given by the expression

$$
\gamma_{1}\left(x_{1}\right)=1-j \sigma\left(x_{1}-x_{n}\right)^{2}
$$

where $x_{n}$ is the coordinate at the interface between the normal domain and the PML and $\sigma$ is a suitable constant. Three different boundary conditions are used for the incoming wave, absorbing boundaries, and inner boundaries:

$$
\begin{gathered}
n_{i}\left(A \frac{\partial u}{\partial x_{i}}\right)-2 j k_{0} \sqrt{A B} u_{0}=0 \quad \text { on } \Gamma_{\text {in }}, \\
n_{i}\left(A \frac{\partial u}{\partial x_{i}}\right)+j k_{0} \sqrt{A B} u=0 \quad \text { on } \Gamma_{\text {out }}, \\
n_{i}\left(A \frac{\partial u}{\partial x_{i}}\right)=0 \quad \text { on } \Gamma \backslash\left\{\Gamma_{\text {in }} \cup \Gamma_{\text {out }}\right\}
\end{gathered}
$$

The unit vector $n_{i}$ is pointing out of the surface. Periodic boundary conditions must be induced for $u$ at the boundaries $\Gamma_{u}$ and $\Gamma_{l}$ (see Fig. 1). When $u_{l}$ represents this quantity on the lower boundary $\Gamma_{l}$ and $u_{u}$ the quantities on the upper boundary $\Gamma_{u}$, then the condition $u_{l}\left(x_{1}\right)=u_{u}\left(x_{1}\right)$ holds. The optical model is solved by the commercial finite element program COMSOL Multiphysics with MATLAB [31]. Here the complex field $u$ is discretized using sets of finite element basis functions $\left\{\phi_{1, n}(\mathbf{r})\right\}$ :

$$
u(\mathbf{r})=\sum_{n=1}^{N} u_{n} \phi_{1, n}(\mathbf{r}) .
$$

The degrees of freedom are assembled in the vector $\mathbf{u}=\left\{u_{1}, u_{2}, \ldots u_{N}\right\}^{T}$. A triangular element mesh is employed, and second-order Lagrange elements are used. This results in the discretized equation

$$
\mathbf{S u}=\mathbf{f},
$$

where $\mathbf{S}$ is the system matrix and $\mathbf{f}$ is the load vector, both being complex valued due to material damping, which is represented by a complex part in $\varepsilon_{r}$. The Si layer $\Omega_{\text {abs }}$ with thickness $a$ absorbs a part of the optical energy, which is calculated by the expression 


$$
\Phi_{H}=\int_{\Omega_{\mathrm{abs}}} \Re\left(-\frac{1}{2}\left[\frac{\partial H_{3}}{\partial x_{1}}\left(\frac{\frac{\partial H_{3}}{\partial x_{1}}}{j \omega \varepsilon_{r}}\right)^{*}+\frac{\partial H_{3}}{\partial x_{2}}\left(\frac{\frac{\partial H_{3}}{\partial x_{2}}}{j \omega \varepsilon_{r}}\right)^{*}\right] \frac{\mathfrak{s}\left(\varepsilon_{r}\right)}{j \varepsilon_{r}}\right) \mathrm{d} \mathbf{r},
$$

for $H_{3}$ polarization. The asterisk indicates complex conjugation. The absorbed energy $\Phi_{E}$ for $E_{3}$ polarization is calculated by the expression

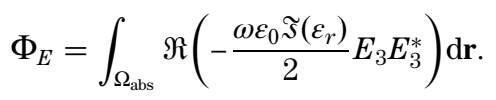

\section{PARAMETER STUDY}

A parameter study of the gap thickness $t$ and width $w$ from Fig. 1 is now performed such that the air gap is optimized for the largest absorption in $\Omega_{\mathrm{abs}}$. This is done for the chosen period $p=500 \mathrm{~nm}$ and for each of the strip materials $\mathrm{Si}, \mathrm{Al}$, $\mathrm{Ag}$, and $\mathrm{Au}$. For the calculations $H_{3}$ polarization is used together with the parameters $a=500 \mathrm{~nm}, \sigma=10^{14} \mathrm{~m}^{-2}$, and the length of the PMLs is $250 \mathrm{~nm}$. The wavelengthdependent dielectric constant $\varepsilon_{r}$ for $\mathrm{Si}$ is taken from [32], and the values for the metals are found in [33]. The optimal values for $t$ and $w$ are found for each of the materials individually for the four increasing target wavelengths $\lambda=440,633$, 849 , and $1051 \mathrm{~nm}$. The absorption in $\Omega_{\text {abs }}$ normalized to the case with a bare surface as a function of the wavelength is plotted in Figs. 2(a)-2(d), where the target wavelength in each case is indicated by the vertical dotted line. In general the structures perform better than a bare surface for the wavelength they are optimized for. For $\lambda=440$ and $633 \mathrm{~nm}$ the case with the $\mathrm{Si}$ strip performs the best and is furthermore better than the metals and the bare surface in almost the entire wavelength interval. For $\lambda=849 \mathrm{~nm} \mathrm{Ag}$ is better than $\mathrm{Si}$, and for $\lambda=1051 \mathrm{~nm} \mathrm{Ag}$ and Au perform significantly better than Si. However, also in the last two cases the optimized Si strips perform well over a broader wavelength interval. Thus, these results indicate that surface structures with $\mathrm{Si}$ can in some cases perform better than structures with metals. Furthermore, structures of Si also seem to perform well for broader intervals in general compared to metals.

In order to explain why Si structures can perform better than plasmonic structures, the absolute value of the magnetic field normalized to the amplitude of the incoming wave $H_{0}$ is plotted for the structures with $\mathrm{Si}$ and $\mathrm{Al}$ in Figs. 3(a) and 3(b), respectively, for the case where they are optimized for $\bar{\lambda}=633 \mathrm{~nm}$. It is seen that the field reaches higher values for the case with the Si strip, and here the wave can also exist within the strip. This helps in building up the mode in $\Omega_{\text {abs }}$, and the absorption is enhanced by $107 \%$ compared to a bare surface. In contrast to that, the wave field can only exist at the border of the metal strip and the enhancement is only $52 \%$ for Al. The logarithm to the absorption $\Phi_{H}$ with the base 10 normalized to the amplitude of the incoming wave is plotted for the same two cases in Figs. 3(c) and 3(d). Here it is seen that more energy is absorbed in $\overline{\Omega_{\text {abs }}}$ in the case with $\mathrm{Si}$ compared to $\mathrm{Al}$, where a significant part of the energy is lost in the $\mathrm{Al}$ strips.

\section{TOPOLOGY OPTIMIZATION}

In the previous section the absorption in $\Omega_{\mathrm{abs}}$ is optimized by a parameter study for the dimensions of rectangular strips on
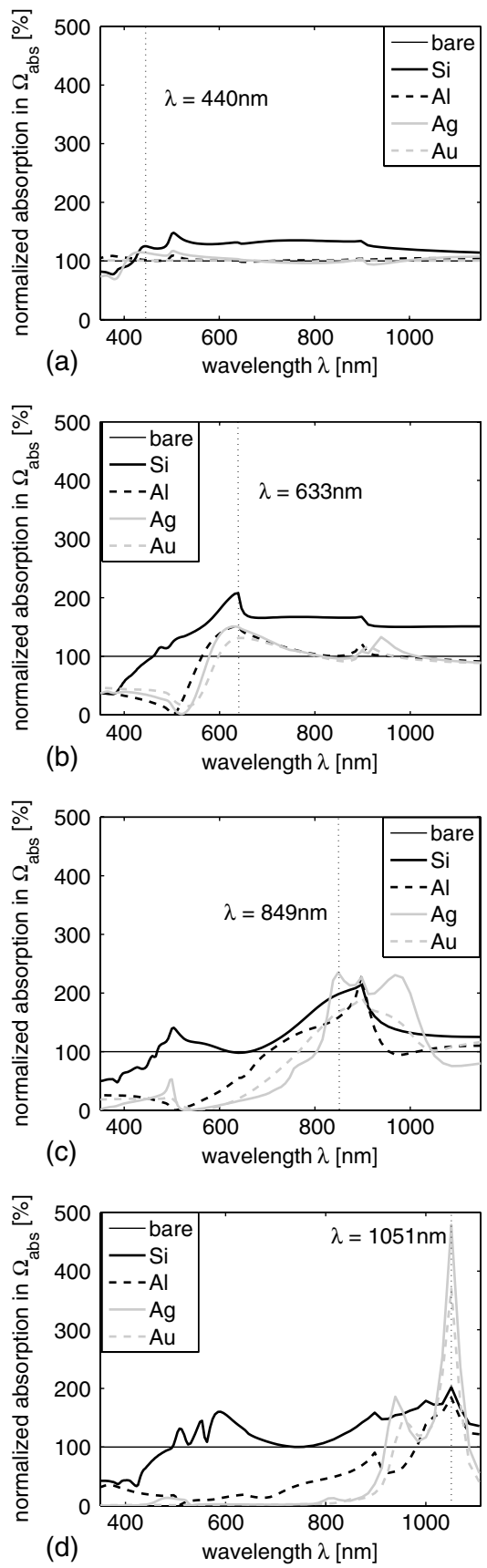

Fig. 2. Normalized absorption in $\Omega_{\text {abs }}$ compared to a bare surface for strips made of the four materials $\mathrm{Si}, \mathrm{Al}, \mathrm{Ag}$, and $\mathrm{Au}$ as function of the wavelength $\lambda$. The four plots are made when the sizes of the strips are optimized for the wavelength, indicated by the vertical dotted line.

the Si surface. A more systematic way to optimize the absorption is to apply the method of topology optimization, where an optimized distribution of air and either $\mathrm{Si}$ or a metal is determined in a design domain $\Omega_{d}$. The structure from Fig. 1 is employed, where $\Omega_{d}$ now constitutes the entire layer at the surface with thickness $t$ and width $p$.

\section{A. Design Variables and Optimization Formulation} Optimizing the material distribution is a discrete problem, as there should be either air or solid material in each point. In order to optimize the problem efficiently, the gradient-based algorithm known as the method of moving asymptotes [34] is 

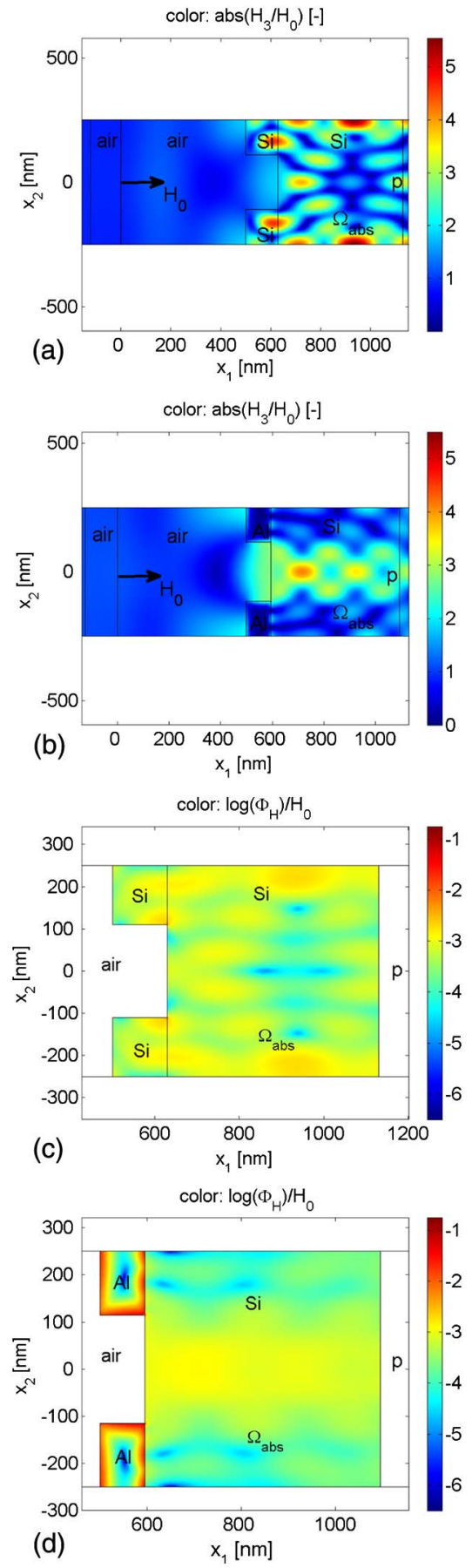

Fig. 3. Results for the structures optimized for $\lambda=633 \mathrm{~nm}$ : the absolute value of the magnetic field normalized to the amplitude of the incoming wave $H_{0}$ for (a) $\mathrm{Si}$ and (b) Al strips; the logarithm to the absorption $\Phi_{H}$ normalized to the amplitude of the incoming wave for (c) Si and (d) Al strips.

employed. Therefore, as is usually done in topology optimization, the problem is formulated with continuous material properties, which can take any value in between the values for air and solid material. Hence, to control the material properties a continuous material indicator field $0 \leq \rho(\mathbf{r}) \leq 1$ is introduced, where $\rho=0$ corresponds to air and $\rho=1$ to solid material. The relative permittivity $\varepsilon_{r}$ is interpolated linearly between the two material phases:

$$
\varepsilon_{r}(\rho)=\varepsilon_{a}+\rho\left(\varepsilon_{s}-\varepsilon_{a}\right) .
$$

In order to get a mesh-independent and discrete $(\rho=0$ or $\rho=1$ ) design, a density filter is employed, where the filter radius is decreased during the optimization. This method also partially counteracts convergence to local optima. The aim of the optimization is to distribute air and solid material in the design domain $\Omega_{d}$ such that the absorption is maximized in the absorbing domain $\Omega_{\text {abs }}$. As $H_{3}$ polarization is used, the optimization problem takes the following form:

$$
\max _{\rho} \log \left(\Phi_{H}\right), \text { objective function }
$$

subject to $0 \leq \rho(\mathbf{r}) \leq 1 \quad \forall \mathbf{r} \in \Omega_{d}$, design variable bounds.

The logarithm is taken to the objective function in order to obtain better numerical scaling for the optimization algorithm.

\section{B. Sensitivity Analysis}

The design variables are updated based on their values from the previous iteration step as well as the derivatives of the objective function with respect to the design variables. In order to calculate these sensitivities, the design variable field $\rho$ is discretized in a similar way as the dependent field:

$$
\rho(\mathbf{r})=\sum_{n=1}^{N_{d}} \rho_{n} \phi_{2, n}(\mathbf{r}) .
$$

The degrees of freedom are assembled in the vector $\boldsymbol{\rho}=\left\{\rho_{1}, \rho_{2}, \ldots \rho_{N_{d}}\right\}^{T}$. Zero-order Lagrange elements are used, and $N_{d}$ is therefore typically smaller than $N$. As is seen from the governing equation [Eq. (7)], the complex magnetic field vector $\mathbf{H}_{3}$ is an implicit function of the design variables. This is written as $\mathbf{H}_{3}(\boldsymbol{\rho})=\mathbf{H}_{3}^{R}(\boldsymbol{\rho})+j \mathbf{H}_{3}^{I}(\boldsymbol{\rho})$, where $\mathbf{H}_{3}^{R}$ and $\mathbf{H}_{3}^{I}$ denote the real and the imaginary part. The derivative of the objective function $\Phi_{H}=\Phi_{H}\left(\mathbf{H}_{3}^{R}(\boldsymbol{\rho}), \mathbf{H}_{3}^{I}(\boldsymbol{\rho}), \boldsymbol{\rho}\right)$ is calculated by the chain rule in the following way:

$$
\frac{d \Phi_{H}}{d \boldsymbol{\rho}}=\frac{\partial \Phi_{H}}{\partial \boldsymbol{\rho}}+\frac{\partial \Phi_{H}}{\partial \mathbf{H}_{3}^{R}} \frac{\partial \mathbf{H}_{3}^{R}}{\partial \boldsymbol{\rho}}+\frac{\partial \Phi_{H}}{\partial \mathbf{H}_{3}^{I}} \frac{\partial \mathbf{H}_{3}^{I}}{\partial \boldsymbol{\rho}}
$$

The derivatives $\partial \mathbf{H}_{3}^{R} / \partial \boldsymbol{\rho}$ and $\partial \mathbf{H}_{3}^{I} / \partial \boldsymbol{\rho}$ are not known directly, as $\mathbf{H}_{3}$ is an implicit function of $\boldsymbol{\rho}$. The sensitivity analysis is therefore performed by employing a standard adjoint method $[20,35]$, where the unknown derivatives are eliminated at the expense of determining an adjoint and complex variable field $\lambda$ from the adjoint equation

$$
\mathbf{S}^{T} \lambda=-\left(\frac{\partial \Phi}{\partial \mathbf{H}_{3}^{R}}-j \frac{\partial \Phi}{\partial \mathbf{H}_{3}^{I}}\right)^{T},
$$

where

$$
\begin{aligned}
\frac{\partial \Phi}{\partial \mathbf{H}_{3}^{R}}-j \frac{\partial \Phi}{\partial \mathbf{H}_{3}^{I}}= & -\int_{\Omega_{o}}\left(\frac{\frac{\partial H_{3}}{\partial x_{1}}}{j \omega \varepsilon_{r}}\right)^{*} \frac{\mathfrak{s}\left(\varepsilon_{r}\right)}{j \varepsilon_{r}} \frac{\partial \phi_{1, n}}{\partial x_{1}} \\
& +\left(\frac{\frac{\partial H_{3}}{\partial x_{2}}}{j \omega \varepsilon_{r}}\right)^{*} \frac{\mathfrak{s}\left(\varepsilon_{r}\right)}{j \varepsilon_{r}} \frac{\partial \phi_{1, n}}{\partial x_{2}} \mathrm{~d} \mathbf{r} .
\end{aligned}
$$


Equation (14) for the derivative then reduces to

$$
\frac{d \Phi}{d \boldsymbol{\rho}}=\frac{\partial \Phi}{\partial \boldsymbol{\rho}}+\Re\left(\lambda^{T} \frac{\partial \mathbf{S}}{\partial \boldsymbol{\rho}} \mathbf{H}_{3}\right)
$$

The vectors $\partial \Phi / \partial \rho$ and Eq. (16), as well as the matrix $\partial \mathbf{S} / \partial \boldsymbol{\rho}$, are assembled in COMSOL Multiphysics as described in [36].

\section{Optimized Designs}

The topology optimization is now performed for several examples with $\lambda=633 \mathrm{~nm}$, and the results are illustrated in Fig. 4 . Two optimized designs are shown for Si in Figs. 4(a) and 4(b) for $t=100$ and $200 \mathrm{~nm}$, respectively, where the white indicates air and the black is solid material. The starting guesses were chosen such that all design variables were equal to 0.5 , and the optimized designs turned out to be discrete. The absorption in $\Omega_{\text {abs }}$ for the optimized designs normalized with the absorption for a bare surface is plotted in Fig. 4(d) as a function of the wavelength $\lambda$. The performance from Fig. 2(b) for the optimized structure from the parameter study in the previous section is indicated as well. The two new designs both perform better for $\lambda=633 \mathrm{~nm}$ than the simpler strip design, where the case for $t=100 \mathrm{~nm}$ performs $117 \%$ better than a bare surface and the case for $t=200 \mathrm{~nm}$ performs $232 \%$ better. In comparison the strip design performed $107 \%$ better than
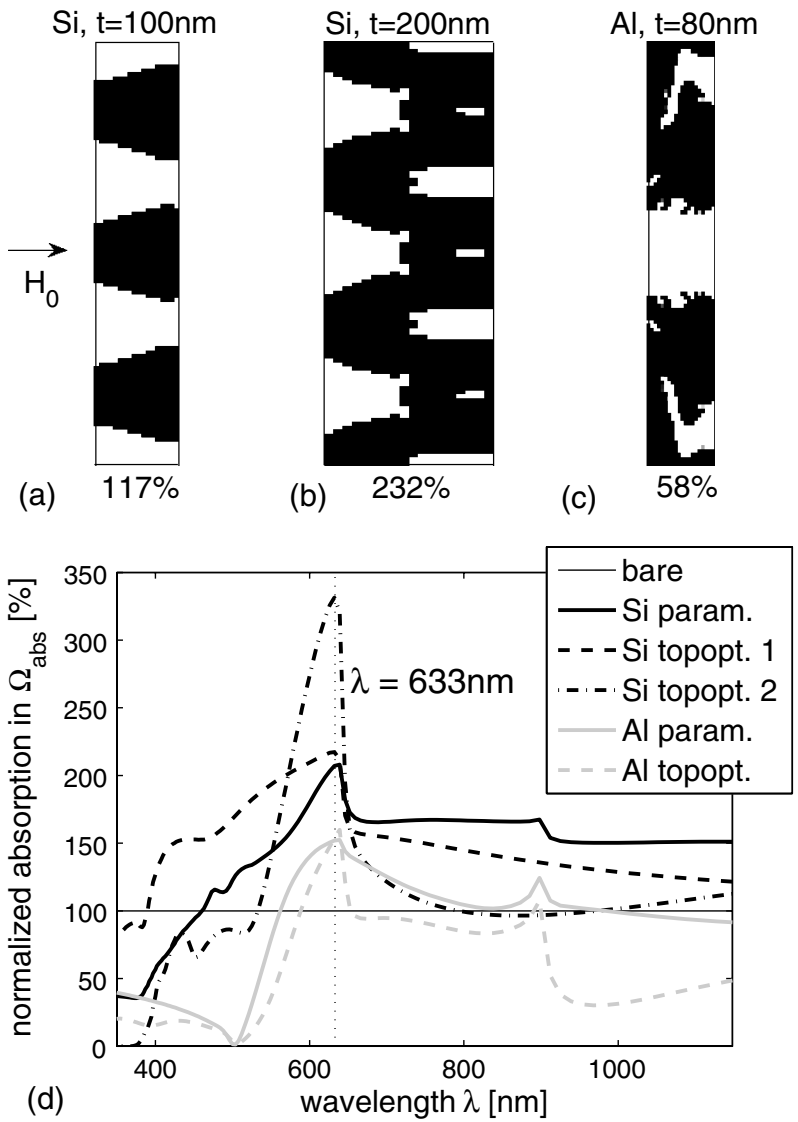

Fig. 4. Results of the topology optimization for $\lambda=633 \mathrm{~nm}$ where white corresponds to air and black to solid material in the optimized designs. (a) Design with Si for $t=100 \mathrm{~nm}$, (b) design with Si for $t=200 \mathrm{~nm}$, (c) design with Al for $t=80 \mathrm{~nm}$, and (d) absorption in $\Omega_{\mathrm{abs}}$ for the optimized designs (topopt) normalized with the absorption for a bare surface as a function of the wavelength $\lambda$. a bare surface for $\lambda=633 \mathrm{~nm}$. This shows that topology optimization is a suitable method to improve the EOA with designs that are nonintuitive and could not be obtained from a simple parameter study. However, the designs from the topology optimization in general have more details, which can be more complicated to fabricate compared to the strip designs. When comparing the two optimized designs, it is seen that the thicker design layer results in an improved performance for the target wavelength, which is a general tendency for increasing thickness, as more material can be used to create greater absorption. This, however, comes at the cost of a worse performance in a broader wavelength interval around the target wavelength.

The topology optimization is also done with $\mathrm{Al}$ in the design domain instead of $\mathrm{Si}$. In this case it is more complicated to obtain designs that perform well, because of the negative real part of the relative permittivity. This property makes it hard to change design variables that are close to air into elements with $\mathrm{Al}$ and vise versa. Therefore, a more clever initial guess must be used compared to $\mathrm{Si}$, where good designs appear for a simple uniform material distribution. The negative permittivity also prevents the designs from performing better for an increasing thickness of $\Omega_{d}$, as the optical wave cannot go through the $\mathrm{Al}$ layer as in the case with $\mathrm{Si}$. The design in Fig. 4(c) is therefore the best that is obtained for a number of different starting guesses and thicknesses. The starting guess here was a gap structure with width $w=100 \mathrm{~nm}$, with design variables equal to 1 outside the gap and 0.9 inside the gap. The thickness is $t=80 \mathrm{~nm}$. The performance improvement of the optimized design is $58 \%$, which is only slightly better than the performance improvement $52 \%$ for the optimized strip design with Al. The design is illustrated in Fig. 4(d). Hence, using topology optimization also indicates that in some cases $\mathrm{Si}$ can increase the EOA more than metals.

The optimization procedure can also be extended to optimize the structure for a sum of the absorptions for a number of wavelengths. An example is presented in Fig. 5, where the optimized design is obtained for the three wavelengths $\lambda=440,633$, and $849 \mathrm{~nm}$, which are indicated in Fig. 5(b) by the vertical dotted lines. Here a uniform material distribution in the design domain with the design variables equal to 0.5 is used as the initial guess and the thickness is $t=150 \mathrm{~nm}$. The optimization results in a design with a better absorption

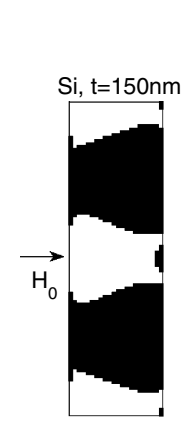

(a)

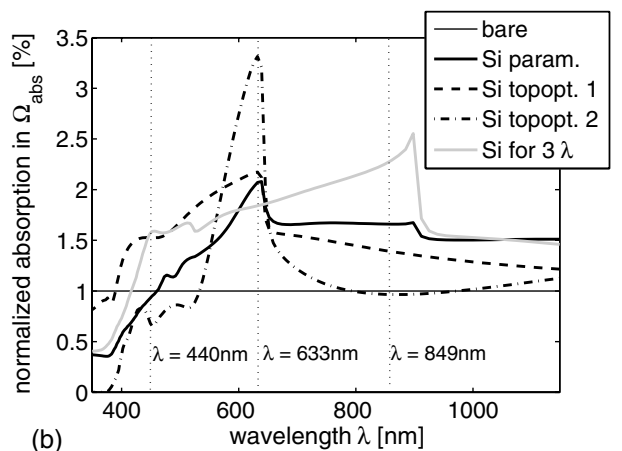

(b)
Fig. 5. Results of the topology optimization for $\lambda=440,633$, and $849 \mathrm{~nm}$. (a) Optimized design with Si for $t=150 \mathrm{~nm}$, where white corresponds to air and black to Si and (b) absorption in $\Omega_{\mathrm{abs}}$ for the optimized designs (topopt) normalized with the absorption for a bare surface as a function of the wavelength $\lambda$. The performance is compared to that of the optimized designs (topopt) from Figs. 4(a) and 4(b). 
for a bigger part of the interval between the three target wavelengths compared to the other three designs obtained for Si [see Fig. 5(b)].

Apart from the topology optimization results shown here, we also experimented with a three-phase version of the software that allows free distribution of three material phases, i.e., void, dielectric, and metallic at the same time. When allowing the optimizer to use free volume fractions, it always ended up in solutions with no metallic content. Metallic designs were only obtained for fixed nonzero volume fractions of metal, and for these structures the objective function values were lower than for purely dielectric designs. This observation clearly demonstrates that for the considered structures, plasmonic effects are undesired. This numerical observation also lead us to the conclusion that purely dielectric strip structures may outperform their plasmonic counterparts, as discussed in [12].

\section{CONCLUSION}

Previous studies have proven that both dielectric and plasmonic structures can cause EOA. Based on two case studies, we show here that it is possible to optimize the EOA using parameter studies and topology optimization of the geometry, as well as by changing the materials.

First, a parameter study is performed where the thickness and the width of strips on top of a Si layer are found for chosen wavelengths in order to increase the absorption in the Si layer. The results are compared for strips made of $\mathrm{Si}$ and the three metals $\mathrm{Al}, \mathrm{Ag}$, and $\mathrm{Au}$. This shows that for some wavelengths the Si strips perform better than the metal strips, and that the optimized structures in general are performing better for a broader wavelength interval.

Second, surface designs of the Si layer are generated with topology optimization. Nonintuitive designs of either air and $\mathrm{Si}$ or air and $\mathrm{Al}$ are obtained, which perform better than the simpler strip designs for a chosen wavelength. For the target wavelength the optimized designs for Si also perform better than the design with Al. This study shows that the method of topology optimization is suitable for obtaining designs that can increase the EOA for a fixed wavelength, and it is also demonstrated that the results can be improved even further by optimizing for a number of wavelengths.

For these particular devices the absorption enhancement is not generated by plasmonic effects but rather is due to confinement of modes related to the surface structuring and the refractive index contrasts. In general we therefore conclude that absorption enhancements for plasmonic structures have to be compared against modulated dielectric structures instead of bare surfaces, so that technologically sensible comparisons can be made.

\section{ACKNOWLEDGMENTS}

This work received support from the Villum Foundation and the Danish Center for Scientific Computing (DCSC).

\section{REFERENCES}

1. T. W. Ebbesen, H. J. Lezec, H. F. Ghaemi, T. Thio, and P. A. Wolff, "Extraordinary optical transmission through subwavelength hole arrays," Nature 391, 667-669 (1998).

2. F. J. G. de Abajo, "Colloquium: light scattering by particle and hole arrays," Rev. Mod. Phys. 79, 1267-1290 (2007).
3. J. N. Anker, W. P. Hall, O. Lyandres, N. C. Shah, J. Zhao, and R. P. Van Duyne, "Biosensing with plasmonic nanosensors," Nat. Mater. 7, 442-453 (2008).

4. J. A. Schuller, E. S. Barnard, W. Cai, Y. C. Jun, J. S. White, and M. L. Brongersma, "Plasmonics for extreme light concentration and manipulation," Nat. Mater. 9, 193-204 (2010).

5. D. Derkacs, S. H. Lim, P. Matheu, W. Mar, and E. T. Yu, "Improved performance of amorphous silicon solar cells via scattering from surface plasmon polaritons in nearby metallic nanoparticles," Appl. Phys. Lett. 89, 093103 (2006).

6. K. R. Catchpole and A. Polman, "Plasmonic solar cells," Opt. Express 16, 21793-21800 (2008)

7. J. S. White, G. Veronis, Z. Yu, E. S. Barnard, A. Chandran, S. Fan, and M. L. Brongersma, "Extraordinary optical absorption through subwavelength slits," Opt. Lett. 34, 686-688 (2009).

8. R. A. Pala, J. White, E. Barnard, J. Liu, and M. L. Brongersma, "Design of plasmonic thin-film solar cells with broadband absorption enhancements," Adv. Mater. 21, 3504-3509 (2009).

9. H. A. Atwater and A. Polman, "Plasmonics for improved photovoltaic devices," Nat. Mater. 9, 205-213 (2010).

10. L. A. Weller-Brophy and D. G. Hall, "Analysis of waveguide gratings: application of Rouard's method," J. Opt. Soc. Am. A 2 863-871 (1985).

11. L. Y. Cao, P. Fan, A. P. Vasudev, J. S. White, Z. Yu, W. Cai, J. A. Schuller, S. Fan, and M. L. Brongersma, "Semiconductor nanowire optical antenna solar absorbers," Nano Lett. 10, 439-445 (2010).

12. M. B. Dühring, N. A. Mortensen, and O. Sigmund, "Plasmonic versus dielectric enhancement in thin-film solar cells," Appl. Phys. Lett. 100, 211914 (2012).

13. O. D. Miller, V. Ganapati, and E. Yablonovitch, "Inverse design of a nano-scale surface texture for light trapping," in CLEO: Science and Innovations, OSA Technical Digest (online) (Optical Society of America, 2012), paper CF2J.2.

14. K. Q. Le, A. Abass, B. Maes, P. Bienstman, and A. Alù, "Comparing plasmonic and dielectric gratings for absorption enhancement in thin-film organic solar cells," Opt. Express 20, A39-A50 (2012).

15. A. Polman and H. A. Atwater, "Photonic design principles for ultrahigh-efficiency photovoltaics," Nat. Mater. 11, 174-177 (2012).

16. M. P. Bendsøe and N. Kikuchi, "Generating optimal topologies in structural design using a homogenization method," Comput Methods.Appl. Mech.Eng. 71, 197-224 (1988).

17. M. P. Bendsøe and O. Sigmund, Topology OptimizationTheory, Methods and Applications (Springer-Verlag, 2003).

18. S. J. Cox and D. C. Dobson, "Maximizing band gaps in twodimensional photonic crystals," SIAM J. Appl. Math. 59, 2108-2120 (1999).

19. O. Sigmund and J. S. Jensen, "Systematic design of phononic band gap materials and structures by topology optimization," Philos. Trans. R. Soc. A 361, 1001-1019 (2003).

20. J. S. Jensen and O. Sigmund, "Topology optimization of photonic crystal structures: a high-bandwidth low-loss T-junction waveguide," J. Opt. Soc. Am. B 22, 1191-1198 (2005).

21. Y. Tsuji, K. Hirayama, T. Nomura, K. Sato, and S. Nishiwaki, "Design of optical circuit devices based on topology optimization," IEEE Photon. Technol. Lett. 18, 850-852 (2006).

22. P. I. Borel, A. Harpøth, L. H. Frandsen, M. Kristensen, J. S. Jensen, P. Shi, and O. Sigmund, "Topology optimization and fabrication of photonic crystal structures," Opt. Express 12, 1996-2001 (2004).

23. J. Riishede and O. Sigmund, "Inverse design of dispersion compensating optical fibres using topology optimization," J. Opt Soc. Am. B 25, 88-97 (2008).

24. M. B. Dühring, O. Sigmund, and T. Feurer, "Design of photonicbandgap fibers by topology optimization," J. Opt. Soc. Am. B 27, 51-58 (2010).

25. K. Fuchi, A. R. Diaz, E. Rothwell, R. Ouedraogo, and A. Temme, "Topology optimization of periodic layouts of dielectric materials," Struct. Multidiscip. Optim. 42, 483-493 (2010).

26. J.A. Andkjær, S. Nishiwaki, T. Nomura, and O. Sigmund, "Topology optimization of grating couplers for the efficient excitation of surface plasmons," J. Opt. Soc. Am. B 27, 1828-1832 (2010).

27. K. S. Friis and O. Sigmund, "Robust topology design of periodic grating surfaces,” J. Opt. Soc. Am. B 29, 2935-2943 (2012). 
28. J. S. Jensen and O. Sigmund, "Topology optimization for nanophotonics," Laser Photon. Rev. 5, 308-321 (2011).

29. U. Basu and A. K. Chopra, "Perfectly matched layers for timeharmonic elastodynamics of unbounded domains: theory and finite-element implementation," Comput. Methods Appl. Mech. Eng. 192, 1337-1375 (2003).

30. R. R. Syms and J. R. Cozens, Optical Guided Waves and Devices (McGraw-Hill, 1992)

31. COMSOL AB, "COMSOL reference manual for COMSOL 3.5, 3.5 edition" (COMSOL AB, Stockholm, Sweden).

32. Virginia Semiconductor, Inc., "Optical properties of silicon," www.virginiasemi.com.
33. A. D. Rakic, A. B. Djurišic, J. M. Elazar, and M. L. Majewski, “Optical properties of metallic films for vertical-cavity optoelectronic devices," Appl. Opt. 37, 5271-5283 (1998).

34. K. Svanberg, "The method of moving asymptotes-a new method for structural optimization," Int. J. Num. Methods Eng. 24, 359-373 (1987).

35. D. A. Tortorelli and P. Michaleris, "Design sensitivity analysis: overview and review," Inverse Probl. Eng. 1, 71-105 (1994).

36. L. H. Olesen, F. Okkels, and H. Bruus, "A high-level programming-language implementation of topology optimization applied to steady-state Navier-Stokes flow," Int. J. Num. Methods Eng. 65, 975-1001 (2006). 